\title{
Simple Analysis Method for the Interrupting Capability of a Contact System in a Molded Case Circuit Breaker
}

\author{
Young-Kil Choi* and Seung-Wook Jee ${ }^{\dagger}$
}

\begin{abstract}
This paper focuses on understanding the interrupting capability of an arc contact system in a molded case circuit breaker (hereafter MCCB). We selected four types of MCCBs and analyzed the magnetic flux density distributions in the contact systems caused by the fault currents. We ascertained that the magnetic flux density profile varies according to the shape of the contact system and was asymmetric at both the ends of an arc, perpendicular to the arc column because of the magnetic grid installed in the contact system. The asymmetric difference creates a magnetic force that pushes the arc current outwards and provides an interrupting capability. We have introduced a simple analysis method for determining the interrupting capability of the contact system for an MCCB by the arcdriving magnetic flux density.
\end{abstract}

Keywords: Arc current, Interrupting capability, Magnetic flux, Contact system, Circuit breaker, MCCB

\section{Introduction}

As various types of electrical equipment and electronic control devices are now being employed as loads, several potential threats such as voltage surges and sudden faults in the power system occur. The widespread dependence on electrical energy has significantly increased with the usage of numerous industrial equipments. In order to assure a reliable power supply, the significance of protective device coordination in electrical cabinet panels has amplified drastically. In a power system with cabinet panels, low voltage circuit breakers are extensively used for interrupting a fault current rapidly and assuring the reliability of the power supply. This increase in market demand has provided the impetus for the related industry to improve the interrupting capability of molded case circuit breakers (hereafter MCCBs) that are one of the low voltage circuit breakers. For an effective cabinet panel, the MCCB should increase the interrupting capability and satisfy the compact requirement for installation. There is a need for standardization of the smaller sized cabinet panels [1-4].

Hence, this paper focuses on understanding the extent of the dependency of the interrupting capability on the shape of the contact system in the MCCB. We have developed a simple analysis method for determining the interrupting capability. First, we chose four types of MCCBs produced by different companies and analyzed the magnetic flux density distributions in their contact systems caused by the fault currents.

We ascertained that the magnetic flux density profile

$\dagger \quad$ Corresponding Author: Dodam Engineering Co., Korea.

(skraken@naver.com)

* Dept. of Small \& Medium Business Support, Korea Electrotechnology

Research Institute (KERI), Korea. (ykchoi@keri.re.kr)

Received: July 20, 2016; Accepted: February 20, 2017 varies according to the contact shape. Some of the contact shapes had a considerably higher flux density. The flux density was asymmetric at both the endpoints of an arc perpendicular to the arc current because of the magnetic grid installed in the contact system. The grid composed of several magnetic plates, is placed in front of the arc current column and it absorbs the flux by the arc on that side only. This creates an unequal flux profile. The asymmetric difference was approximately equal to the increase in the interrupting capability because it created a magnetic force and pushed the arc current towards the grid and outwards.

The arc column ceases to be straight and becomes a longer curve. The longer the arc length owing to the arc curve, the higher is the resistance. As a result, the interrupting capability is improved.

Thus, we have introduced a simple analysis method for checking the extent of the interrupting capability by the asymmetric magnetic flux driving the arc current.

\section{Simple Analysis Method for the Interrupting Capability of the Contact System}

As the arc between the two contacts in the MCCB's contact system remains in a plasma state near current zero point [3], this arc can be easily quenched if a strong asymmetric magnetic flux is formed around it. In order to form this magnetic flux, several researchers have tried various types of magnetic grids and have improved the interrupting capability of the MCCB. In the experiment in [5], however, while the interrupting current is increased, the arc-driving magnetic flux formed by the grid, is unable to increase beyond a certain range because of the magnetic saturation phenomenon. For solving this problem, several 


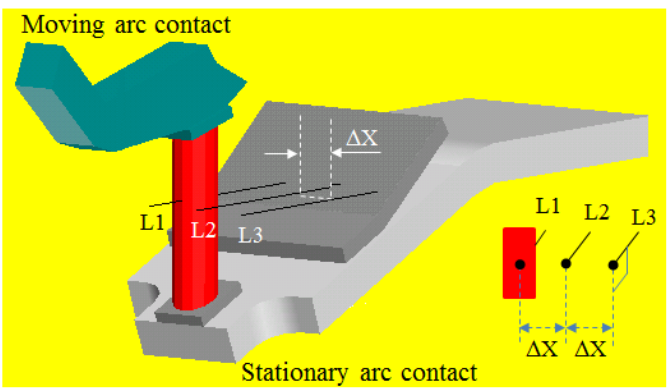

Fig. 1. Three analytical lines for the magnetic flux density distribution profile

efforts have been made to form a strong arc-driving magnetic flux along with the current. Some researchers have suggested that improving the shape of the contact system could result in the formation of a strong arc-driving magnetic flux [6]. As the magnetic flux formation increases along with the interrupting current, the current flow needs to be effectively utilized. Hence, the contact shape has to be designed accordingly. A suitable shape can concentrate the flux density in a certain place to get stronger and more asymmetric. Therefore, we attempted a 3D flux analysis for the magnetic flux distributions in different contact systems. This step needs to be undertaken prior to design of a new contact system for better results.

First, we chose four types of MCCBs produced by different manufacturers. The product specifications along with the rated interrupting current are displayed on the MCCBs. Next, we illustrated the shapes of the contact systems and analyzed them in 3D. From the analysis results, we were able to determine the magnetic flux density distributions. After the calculations for the analysis, we selected three analytical lines to realize the distribution profile, as shown in Fig. 1. Line L1 is a base line that passes through the center of the arc column; line L2 is at a distance $\Delta X$ from the arc column and line L3 is at a distance $2(\Delta X)$. We set up the arc as a round, as in Fig. 1 and formed the analytical lines at certain heights each from the bottom of the stationary arc contact.

Then, we determined the values of the flux densities on these three lines. We plotted a graph depicting the flux densities generated by the four types of contact systems and compared their interrupting capabilities.

\subsection{Conditions for simple analysis method}

Some papers $[6,7]$, report the effects of the magnetic flux distribution on a contact system with an interrupting current. We have developed a simple analysis method for understanding the interrupting capability and the role of the arc-driving magnetic flux density on the shape of the contact system. In this paper, the following conditions are considered.

- Arc radius: $2 \mathrm{~mm}$

- Interrupting current : $25 \mathrm{kA}$

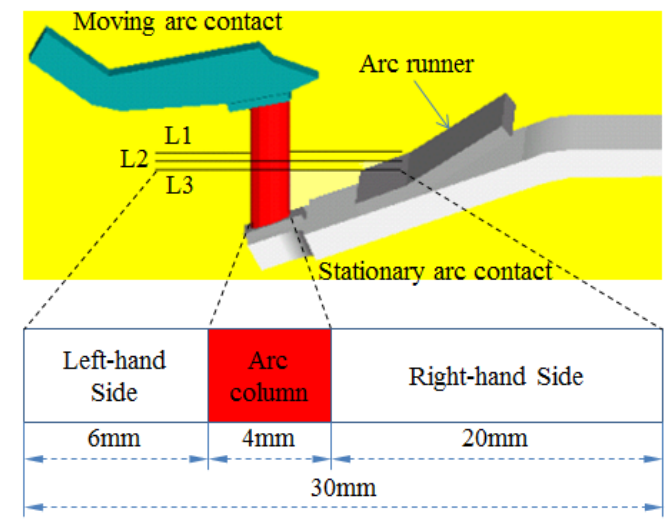

Fig. 2. Condition and arrangement for the simple analysis method

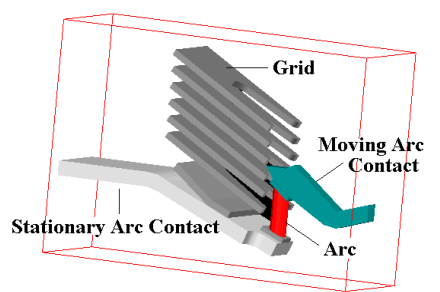

(a)

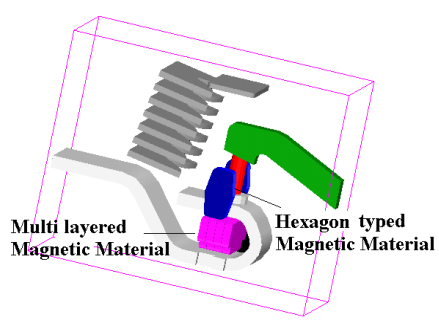

(c)

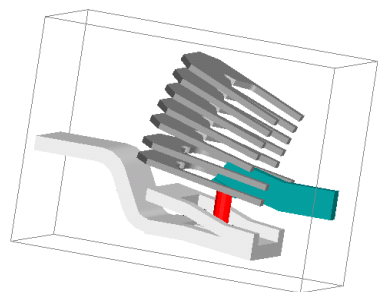

(b)

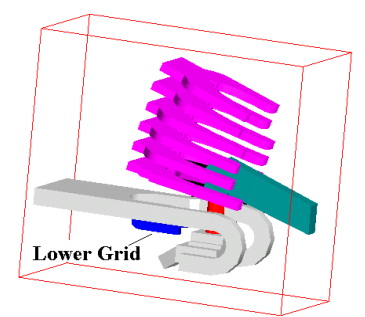

(d)
Fig. 3. 3D shapes of the contact systems for the magnetic flux analysis: (a) MCCB made by the sponsoring domestic company (model name : M1); (b) MCCB made by another domestic company (M2); (c) MCCB made by an European company (M3); (d) MCCB made by a Japanese company (M4)

- Distance between the two contacts : $8 \mathrm{~mm}$

- Arc column : cylinder in a straight line

Using the arc column as the center, the moving contact part is located on the left and the stationary arc contact is on the right.

\subsection{Characteristics of the shape of the contact system}

Fig. 3 exhibits the various contact systems used for the magnetic flux analysis. Two of the MCCBs are manufactured by domestic companies. The first (M1) in Fig. 3(a) is made by the sponsoring domestic company that participated in this research and the second (M2) in Fig. 3(b) is made by another domestic company. The other two MCCBs are produced by foreign companies. The third 
(M3) in Fig. 3(c) is made by a European company and fourth (M4) in Fig. 3(d) is made by a Japanese company. All these products are presently available in the market. All the selected model breakers had a rated current of $225 \mathrm{~A}$. However, the rated interrupting current varied. An isolation cover is at times employed at the head of the stationary arc contact with the arcing spot. It is useful in continuously maintaining the initial spot of the arc column root and preventing it from shortening the arc length because the arc moves and its root point can change to another spot along the stationary arc contact path. Models M2 and M4 had isolation covers. In model M3, the stationary arc contact is shaped like the character ' $\mathrm{J}$ ' and a hexagonal magnetic material is used both to the left and right of the contact system.

It appears to gather the magnetic flux density, enabling it to drive the arc. In addition, a multi-layered magnetic material is placed under the stationary arc contact. Its purpose may be to absorb the magnetic flux against the arc drive. In M4, the stationary arc contact is bent into a halfcoil shape for concentrating the magnetic flux inside and a sub-grid is placed within the stationary arc contact.

\subsection{Simple magnetic analysis of the contact system}

The direction of the magnetic force is given by the right hand rule as shown in Fig. 4 and the magnetic force relationship is in the form of a vector product as in (1).

$$
\vec{F}=\left(\vec{I} \int_{0}^{2 \pi} \vec{B}\right) l_{\text {arc }}
$$

where, the arc length is $l_{\text {arc }}$, the vector arc current is $\vec{I}$, the vector magnetic flux density is $\vec{B}$, and the arc driving force is $\bar{F}$.

When (1) is applied to the arc current, the right-hand rule may be used to determine the direction of the force on the arc. In Figs. 4 and 5, the magnetic flux density $\mathrm{B}(\mathrm{L})$ on the left forms a force $\mathrm{F}(\mathrm{L})$ that drives the arc, pushing it from left to the right.

On the other hand, the magnetic flux density $\mathrm{B}(\mathrm{R})$ on the right forms $F(R)$ opposite to $F(L)$. The density is the same

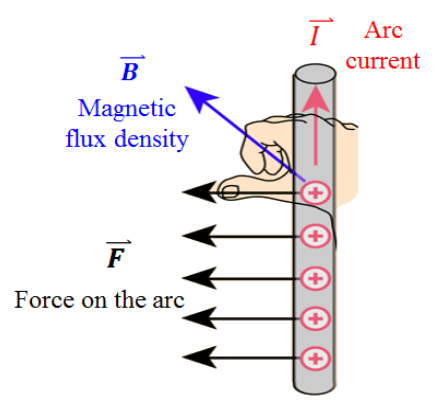

Fig. 4. The magnetic force relationship in the form of a vector product and it is well balanced in all aspects; the total force is zero as in (1).

However, the magnetic grid with several plates in the contact system absorbs the flux lines on the right and the arc- driving force $F(L)$ remains as such but the force $F(R)$ becomes considerably smaller than $F(L)$. The force balance finally becomes asymmetric $\mathrm{F}(\mathrm{L})>\mathrm{F}(\mathrm{R})$. This asymmetric condition causes the arc to move to the right, extending the arc and interrupting the arc current. Extending the arc implies that the arc resistance $R_{\text {arc }}$ becomes infinite, as shown in (2).

$$
R_{\text {arc }}=\frac{l_{\text {arc }}}{\sigma_{\text {arc }} A_{\text {arc }}}
$$

if $R_{\text {arc }} \rightarrow \infty$, the current is interrupted.

where, the arc resistance is $R_{\text {arc }}$, the electrical conductivity is $\sigma_{\text {arc }}$, and the cross section of the arc is $A_{\text {arc }}$, respectively. The longer the arc length, the larger is the resistance and the easier the current interruption. It is to be noted that the resistance $R_{\text {arc }}$ is dependent and proportional to the arc length $l_{\text {arc }}$. In order to obtain the difference $\Delta \mathrm{B}$ between $\mathrm{B}(\mathrm{L})$ and $\mathrm{B}(\mathrm{R})$, a $3 \mathrm{D}$ magnetic analysis is done by applying the conditions set in 2.1 . It enables the evaluation of the interrupting capability of the contact systems.

We first examined the density difference $\mathrm{B}_{P 1-P 2}^{L_{1}}$ on the line L1 at both the endpoints of the arc from side to side, as follows :

$$
\mathrm{B}_{P 1-P 2}^{L_{1}}=\mathrm{B}(\mathrm{L})_{P 1}^{L_{1}}-\mathrm{B}(\mathrm{R})_{P 2}^{L_{1}}
$$

where $\mathrm{B}(\mathrm{L})_{P 1}^{L_{1}}$ is the magnetic flux density at a point $P_{1}$ on the left and $\mathrm{B}(\mathrm{R})_{P 2}^{L_{1}}$ is the magnetic flux density at a point $P_{2}$ on the right, as shown in Fig. 5.

When we compared the difference $\mathrm{B}_{P 1-P 2}$ on the analytical line L1 for all the models (Fig. 6), we found that it was the largest for model M4, followed by M3, M2, and M1.

Almost similar trends were found for the density differences, $\mathrm{B}_{P 1-P 2}^{L_{2}}$ and $\mathrm{B}_{P 1-P 2}^{L_{3}}$ on the analytical line $\mathrm{L} 2$. The value $\mathrm{B}(\mathrm{L})_{P 1}^{L_{1}}$ shows that most of models had a value more than 3.0 T, as shown in Fig. 6. All the models except $\mathrm{M} 1$ recorded levels under approximately $0.5 \mathrm{~T}$ in the $15-20$ $\mathrm{mm}$ range of the analytical lines. We consider that this low

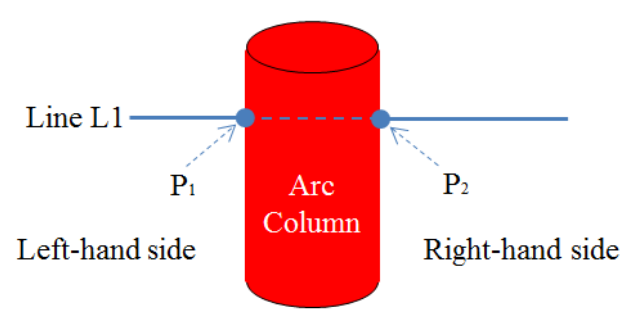

Fig. 5. Both the ends of the arc on the analytical line, L1 


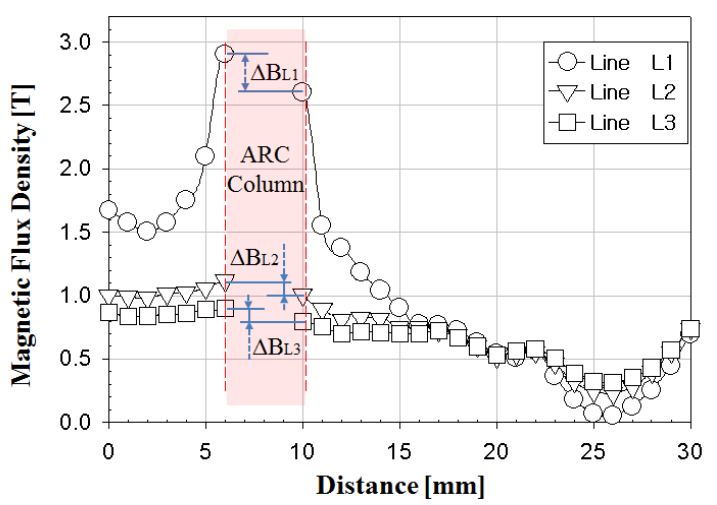

(a) Result for model, M1

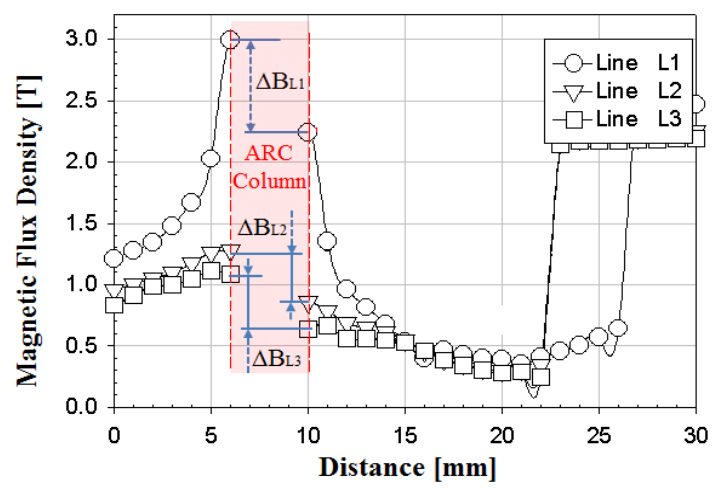

(c) Result for model, M3

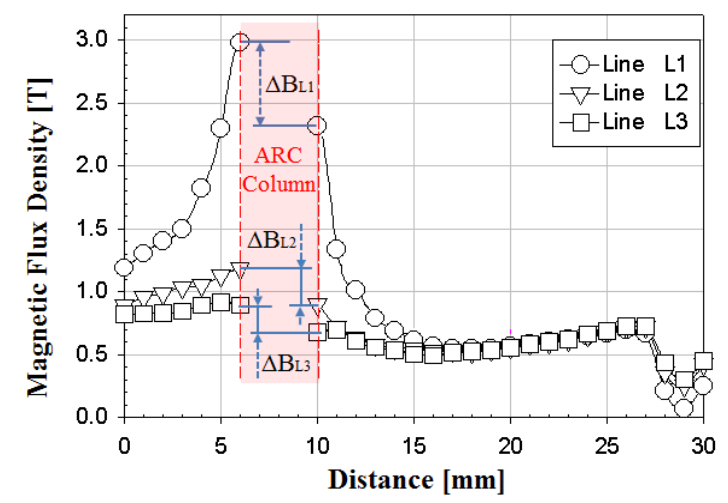

(b) Result for model, M2

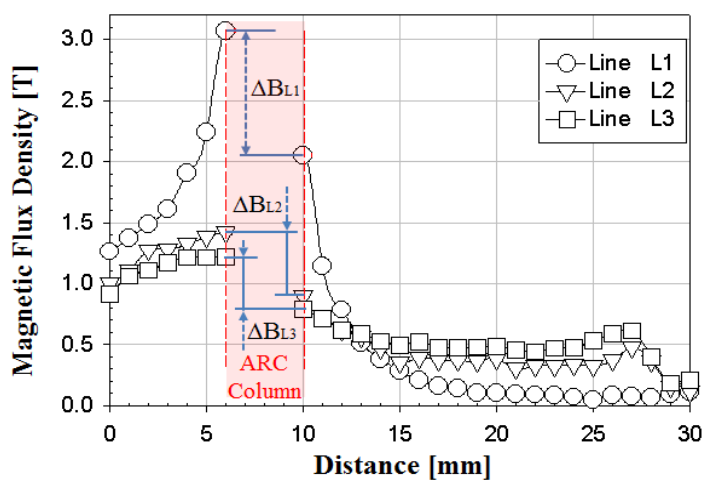

(d) Result for model, M4

Fig. 6. Results for the 3-D magnetic flux analysis on the analytical lines

Table1. Results of the 3D magnetic flux analysis

\begin{tabular}{c|c|c|c|c}
\hline \multirow{2}{*}{ Model } & \multicolumn{3}{|c|}{$\begin{array}{c}\text { Difference of the magnetic flux } \\
\text { densities at both endpoints of the arc }\end{array}$} & \multirow{2}{*}{$\begin{array}{c}\text { Rated Interrupting } \\
\text { Current }\end{array}$} \\
\cline { 2 - 4 } & $\mathrm{B}_{P 1-P 2}^{L_{1}}$ & $\mathrm{~B}_{P 1}^{L_{2}}-P 2$ & $\mathrm{~B}_{P 1-P 2}^{L_{3}}$ & \\
\hline M1 & 0.30 & 0.13 & 0.12 & $\mathrm{AC} 460 \mathrm{~V} / 25 \mathrm{kA}$ \\
\hline $\mathrm{M} 2$ & 0.70 & 0.30 & 0.20 & $\mathrm{AC} 460 \mathrm{~V} / 35 \mathrm{kA}$ \\
\hline $\mathrm{M} 3$ & 0.77 & 0.42 & 0.48 & $\mathrm{AC} 440 \mathrm{~V} / 65 \mathrm{kA}{ }^{(*)}$ \\
\hline M4 & 1.00 & 0.50 & 0.45 & $\mathrm{AC} 440 \mathrm{~V} / 50 \mathrm{kA}$ \\
\hline Note(*) & The Model, M3, has the rotary contact system with dual contacts/ \\
phase
\end{tabular}

value can contribute better to the fault current interruption. Because the magnetic force $\mathrm{F}(\mathrm{L})$ drives the body of the arc column far away from the arc root spot above the magnetic grid, it increases the resistance $R_{\text {arc }}$ raising the arc voltage also.

Summarizing the above-mentioned contents in Table 1, it is considered necessary to design the shape so that the contact system concentrates the magnetic flux density in a certain place, in the same direction, and renders the magnetic force balance asymmetric using the magnetic grid.

As a result, the model M4 is the best model among four types with the detailed reason.

\section{Conclusion}

In the event of an extremely high overcurrent condition (e.g., a short circuit), the operating mechanism in the MCCB repels the moving arc contact away from the stationary arc contact. Once activated, the operating mechanism separates the two contacts to stop the flow of current in the protected circuit. In order to accommodate the solutions for the various fault conditions that can occur within an electrical distribution system, efficient MCCBs are required based on shapes that are suitably designed for the fault current interruption.

For determining the effectiveness of the designed shape, we developed a simple analysis method to understand and estimate the interrupting capability of the contact system for which the MCCB shape is designed. Using three analytical lines in a simple analysis method, we calculated the magnetic flux density distributions on the lines for four models of MCCBs. The differences in the flux densities at both the endpoints of the arc, side to side, for all the models were determined. A considerably large value was expected to provide a better interrupting capability. It was determined that the fault current interrupting capability increases almost proportionally to the magnitude of the arc-driving magnetic flux density. 
Thus, the simple analysis method enables an understanding of the performance of the MCCB for achieving an optimal shape, before starting the actual production.

\section{References}

[1] Jong-Suk Ro, Hyeon-Jeong Bak, Hyun-Kyo Jung, "Characteristic Analysis and Design of a Novel Lorentz Force Driving Actuator for a Molded Case Circuit Breaker," IET Electr. Power Appl., Vol. 9, Iss.1, pp. 1-9, 2015.

[2] Li, X., Chen, D., Wang, Y., Wang, Q., Geng, Y., "Analysis of the Interruption Process of Molded Case Circuit Breakers," IEEE Trans. Compon. Packag. Technol., vol. 30, no. 3, pp. 375-382, Sep. 2007.

[3] John J. Shea, Xin Zhou, "Contact Material and Arc Current Effect on Post-Current Zero Contact Surface Temperature," IEEE Trans. Components and Packaging Technologies, vol. 29, no. 2, pp. 286-293, Jun. 2006.

[4] David D. Roybal, "Circuit-breaker Ratings : Weighing High Interrupting Capacity and Short-time Current Ratings," IEEE Industry Applications Magazine, pp. 61-71, Jul/Aug. 2005.

[5] Mitsugu Takahashi, Takao Mitsuhashi, Yuuichi Wada, Sinji Yamagata, "Building up the Arc Driving Magnetic Flux in the Contact System of MCCB," SP-95-56 Published by Japanese Institute of Electrical Engineers, pp. 171-180, 1995.

[6] Special Issue, "New Circuit Breakers \& Earth Leakage Circuit Breakers," Mitsubishi Denki Giho, vol. 69 , no. 4, 1995.

[7] Special Issue, "New MCCB, ELCB," Fuji Electric Journal, pp. 124-177, 1990.

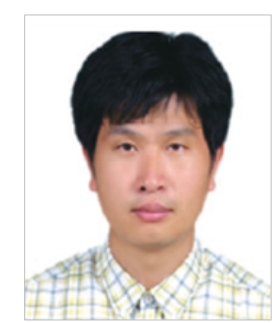

Young-Kil Choi He received his $\mathrm{PhD}$ degree in Electrical Engineering from Yeungnam University, Korea in 2012. In 1993, he joined the Korea Electrotechnology Research Institute (KERI), Korea, His research interests are power circuit breaker simulation and design.

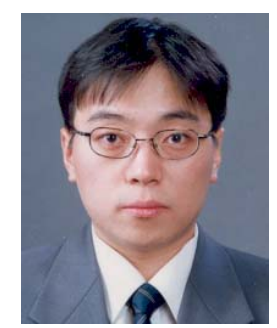

Seung-Wook Jee He received the B.E. M.E. and Ph.D. degrees in electrical engineering from the Yeungnam University, Korea, in 1995, 1997, and 2005, respectively. His research interests are electrical safety and fire alarm system. $\mathrm{He}$ is currently working in the field of fire detection. 